\title{
Hyperbaric oxygen therapy and surgical delay improve flap survival of reverse pedicle flaps for lower third leg and foot reconstruction
}

\author{
Pradeoth Mukundan Korambayil, Prashanth Varkey Ambookan \\ Department of Plastic Surgery and Burns, Jubilee Institute of Surgery for Hand, Aesthetic and Microsurgery, Jubilee Mission Medical \\ College and Research Institute, Thrissur 680005, Kerala, India.
}

Address for correspondence: Dr. Pradeoth Mukundan Korambayil, Department of Plastic Surgery and Burns, Jubilee Institute of Surgery for Hand, Aesthetic and Microsurgery, Jubilee Mission Medical College and Research Institute, Thrissur 680005, Kerala, India. E-mail: pradeoth@gmail.com

\begin{abstract}
Aim: The purpose of the study is to present a management protocol for various types of soft tissue defects of the distal third region of leg and foot treated with pedicle flaps, by including hyperbaric oxygen $(\mathrm{HBO})$ therapy in the treatment regimen with flap delay. Methods: We present a prospective study of 23 patients with various types of soft tissue defects of the foot, and lower third of leg managed in our institution from December 2012 to December 2013. All soft tissue defects were treated by a reverse pedicle flap. Twelve patients were managed with flap delay with $\mathrm{HBO}$ therapy and 11 patients with immediate flaps without $\mathrm{HBO}$ therapy. The postoperative period, hospital course, and follow-up were documented. Results: Of 12 patients with flap delay and HBO, 10 patients did not suffer any complications secondary to flap transfer. One patient had discoloration of the tip of the flap, which settled without the intervention, and 1 patient had recurrent abscess formation, which required debridement and closure. Of 11 patients with direct transfer, 6 patients presented with complications including flap congestion, partial flap loss, and tip necrosis, which required secondary intervention. Conclusion: HBO therapy is a useful adjunct in flap delay of the reverse pedicle flap for soft tissue reconstruction of the lower third of the leg and foot regions.
\end{abstract}

Key words:

Flap delay, hyperbaric oxygen therapy, reverse pedicle flap, soft tissue reconstruction

\section{INTRODUCTION}

Although numerous techniques ranging from skin grafting to free-tissue transfer have been utilized for reconstruction of soft tissue defects of the foot, and lower third of the leg regions, very few have yielded entirely satisfactory results. A safe, easy and reliable reconstructive option is required for reconstruction of

\begin{tabular}{|l|l|}
\hline \multicolumn{2}{|c|}{ Access this article online } \\
\hline Quick Response Code: & Website: \\
\hline & www.parjournal.net \\
\cline { 2 - 3 } & \\
\hline & DOI: \\
\hline
\end{tabular}

the lower third of the leg and foot regions. The lateral supramalleolar flap ${ }^{[1,2]}$ from the lateral aspect of the lower leg, and the flap supplied by a perforating branch of the dorsal peroneal artery are commonly used for coverage of the dorsum of the foot, the medial and lateral arches, and all regions of the heel. The reverse sural flap $^{[3,4]}$ is raised from the posterior aspect of the calf and is commonly used for coverage of the hind foot, dorsum, and the lateral malleolus. Distally-based flaps based on the posterior tibial artery or the peroneal artery perforator plus flap ${ }^{|5|}$ also assist in the soft tissue coverage of defects of the distal third of the leg. In this prospective study, 23 patients were treated with distally-based pedicle flap coverage in which 12 patients underwent flap delay with hyperbaric oxygen (HBO) therapy, and 11 patients underwent immediate flap transfer without HBO therapy. The study was undertaken prospectively over a period of 
13 months in a tertiary care unit. The purpose of this study was to present a management protocol for various types of soft tissue defects of the distal third of the leg and foot with pedicle flaps, by including HBO therapy in the treatment regimen with the flap delay. The study was approved by the review board of Jubilee Mission Medical College and Research Institute.

\section{METHODS}

A total of 23 patients with soft tissue defects of lower third of the leg and foot were treated over a period of 13 months (December 2012 to December 2013). On the basis of the defects, lateral supramalleolar, reverse sural and distally-based posterior tibial artery perforator plus flaps were utilized for soft tissue coverage. Of 23 patients, 12 patients were managed by flap delay with HBO therapy as an adjunct, and 11 patients were managed by direct flap transfer without HBO therapy. Outcomes following the different types of management and secondary procedures performed were noted. All involved patients gave their consent forms.

\section{RESULTS}

The mechanism of injury for 20 patients was a road traffic accident, 1 patient presented with an open wound secondary to a diabetic ulcer, 1 defect was due to osteomyelitis and 1 was due to a snake bite injury. Of 23 patients, there were 6 females (26.1\%) and 17 males patients $(73.9 \%)$. The mean age was 42 years (range: 13-68 years).

In 12 patients, the flap delay was performed, and HBO therapy was used as an adjunct. Of these 12 patients, 2 patients had sustained the defect due to an infectious source, and 10 cases occurred secondary to a road traffic accident. Among these 12 patients, 5 patients had diabetes mellitus, and 1 patient had soft tissue loss due to a snake bite injury. Two patients were scheduled for extended sural artery flap, and 1 patient had varicose veins as a comorbid condition. Five flaps were lateral supramalleolar flaps, 1 was a distally-based posterior tibial artery perforator flap, and 6 were reverse sural artery flaps. Among the 6 reverse sural flaps, 2 were extended reverse sural flaps. The severity of injury, time of referral, comorbid conditions, age of the patients, and the extent of the flap were considered to be qualifying conditions for flap delay with associated HBO therapy. One patient developed a recurrent abscess at the ankle joint, which required incision and drainage, and 1 patient had discoloration of the flap tip which resolved without intervention.

For 11 patients, direct transfer of the flap was performed. Of these 11 patients, 1 child had suffered soft tissue loss secondary to osteomyelitis, and the rest of the defects were due to road traffic accidents. Four flaps were lateral supramalleolar flaps and 7 were reverse sural artery flaps. Six patients developed postoperative complications. Five patients suffered tip necrosis, which required debridement and skin grafting, and 1 patient sustained partial loss of a reverse sural flap, which subsequently required skin grafting [Tables 1-3].

\section{Case I}

A 34-year-old male with a crush injury to the right heel pad and ankle region was referred to our center 3 weeks following injury. The patient presented with necrosis of the heel pad with multiple lacerations over the ankle on both the medial and lateral aspects [Figure 1a-c]. The wound was debrided, and HBO therapy sessions were started. On postdebridement day 2, an extended reverse sural flap was elevated with flap delay [Figure 1d], continuing the hyperbaric sessions. On postdebridement day 4, flap inset was completed [Figure 1e]. The donor area was covered with a split-thickness skin graft. HBO therapy was administered for an additional 12 sessions. The postoperative period was uneventful [Figure 1f and g].

\section{Case 2}

A 68-year-old male developed an ulceration on the lateral malleolus with exposure of the ankle joint [Figure 2a]. HBO therapy sessions were started. The wound was debrided, and lateral supramalleolar flap coverage was planned [Figure 2b]. The lateral supramalleolar flap was elevated, and flap delay was performed, with continuation of the hyperbaric sessions [Figure 2c]. On postdebridement day 4, flap inset was completed, and the donor area was covered with a split-thickness skin graft [Figure 2d]. HBO therapy was administered for 12 additional sessions. The postoperative period was uneventful [Figure 2e].

\section{Case 3}

A 21-year-old male sustained injury to the lower third of the right leg with soft tissue loss and exposure of the tibial bone [Figure 3a]. Following debridement, a distally-based posterior tibial artery perforator plus flap was planned. A distally-based posterior tibial artery perforator plus flap was elevated, and flap delay was performed with continuation of the hyperbaric sessions [Figure $3 b$ and $c$ ]. On postdebridement day 4, flap inset was completed, and the donor area was covered with a split-thickness skin graft [Figure 3d]. HBO therapy was administered for an additional 6 sessions. The postoperative period was uneventful [Figure 3e].

\section{Case 4}

A 39-year-old male sustained injury to the right foot secondary to a road traffic accident and presented with soft tissue loss over the medial malleolar and calcaneal regions [Figure 4a]. The wound was debrided, and a reverse sural artery flap was performed [Figure $4 \mathrm{~b}$ and $\mathrm{c}$. The patient developed flap tip necrosis and required a skin graft for coverage [Figure $4 \mathrm{~d}$ ].

\section{DISCUSSION}

Soft tissue defects of the lower third of the leg and foot may be covered with skin grafts, local flaps, distally or proximally-based island flaps, and distant tissue transfer or cross leg flaps. In 1983, distally-based fasciocutaneous flaps were introduced, providing flaps with a reliable vascular supply regardless of their length to width ratio. The reverse sural artery flap, lateral supramalleolar flap, and inferiorly-based medial and lateral fasciocutaneous flaps 


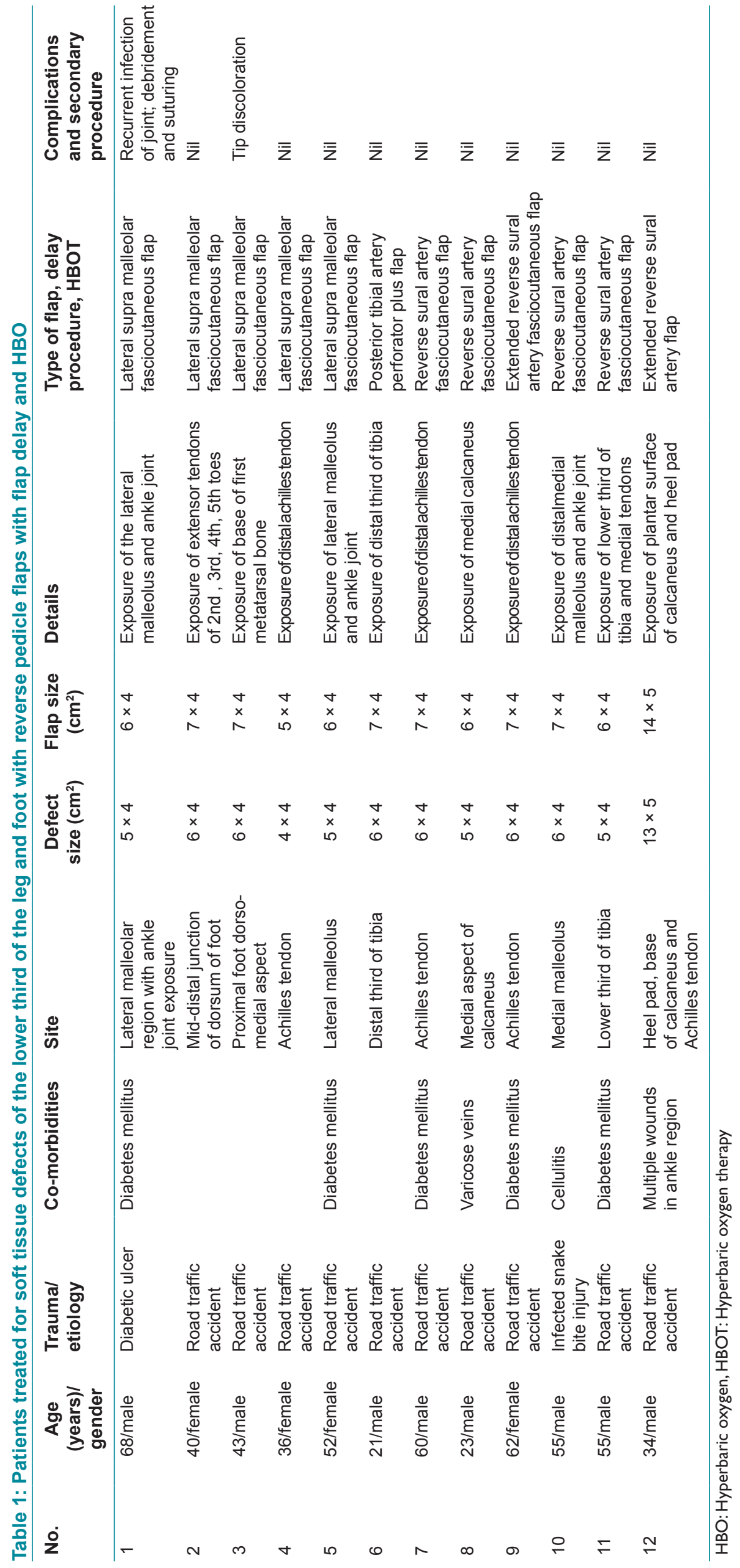




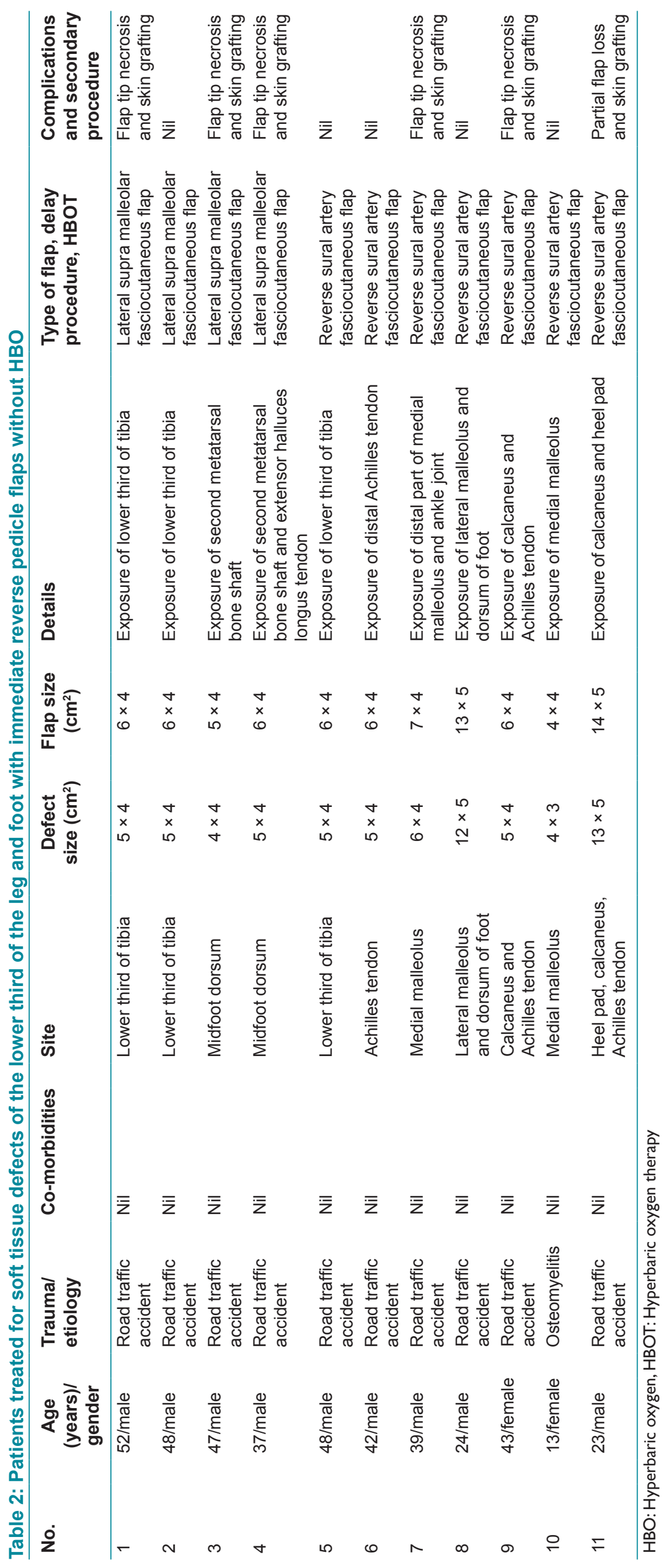




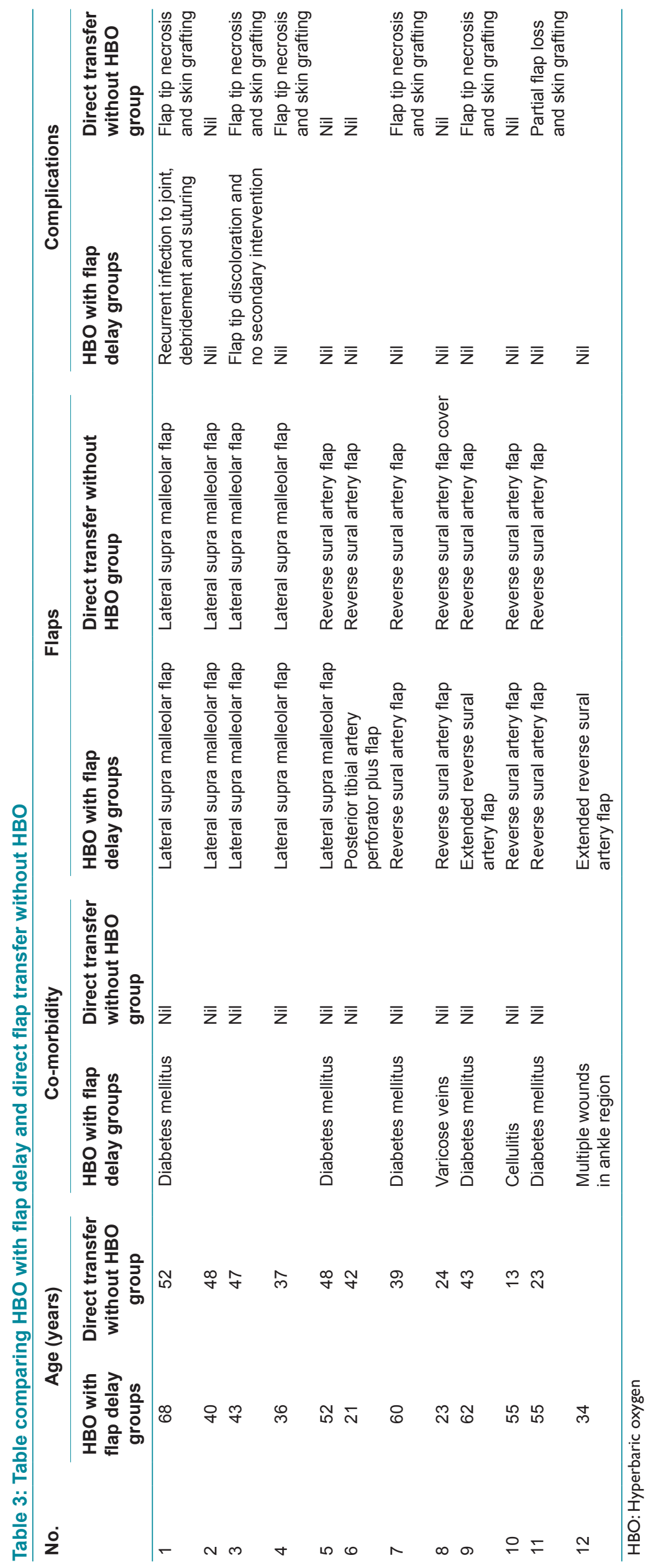




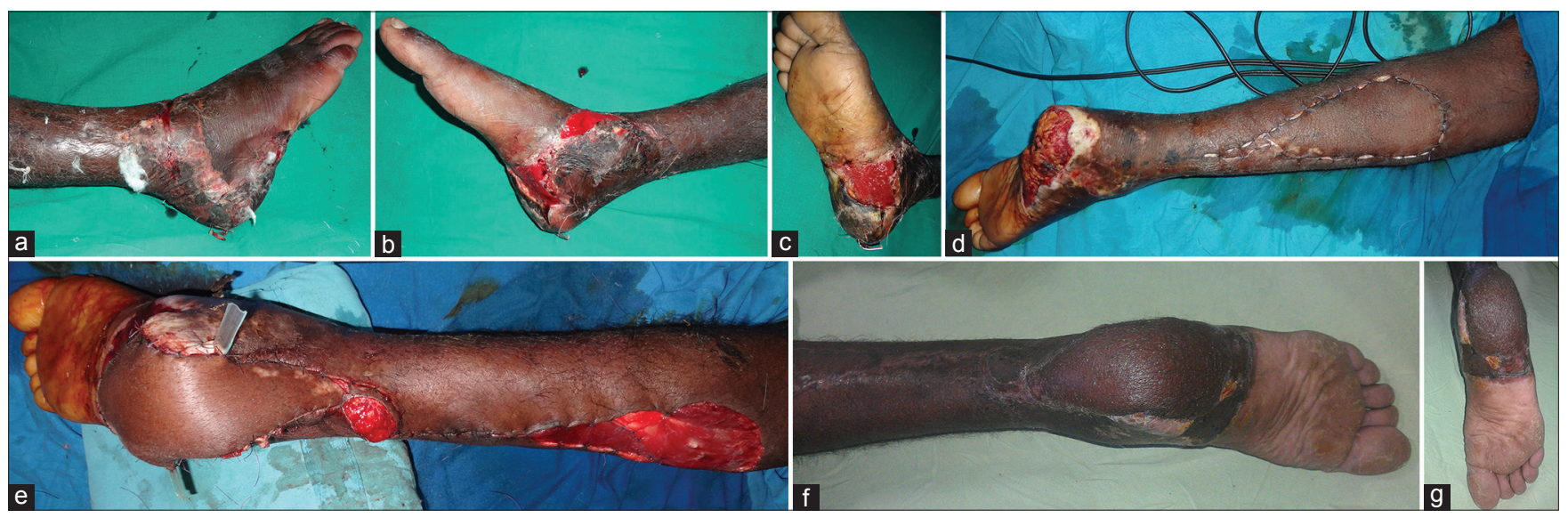

Figure 1: (a) Crush injury to the right heel pad and ankle regions with multiple lacerations on the medial aspect of the foot; (b) crush injury to the right heel pad and ankle regions with multiple lacerations on the lateral aspect of the foot; (c) picture showing necrotic heel pad tissue; (d) extended reverse sural artery flap was elevated on day 2 following debridement; (e) extended reverse sural artery flap delay performed; (f) flap inset was completed on day 4 following debridement; (g) postoperative day 21 following surgery-posterior view

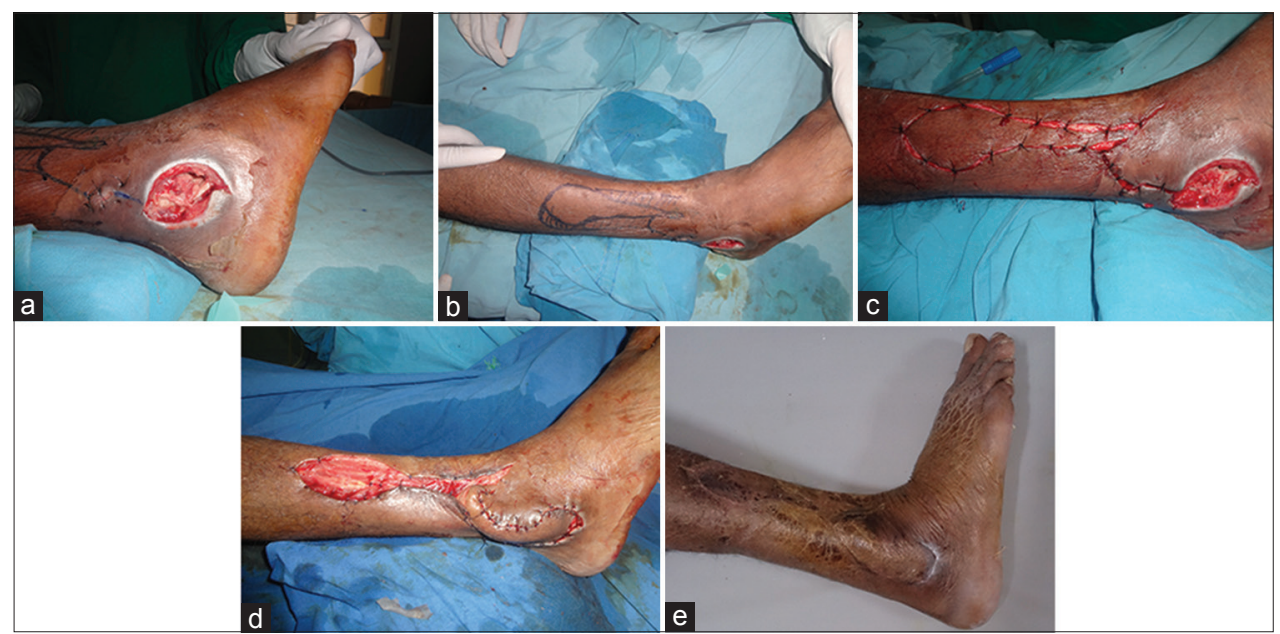

Figure 2: (a) Ulceration on the lateral malleolus with exposure of the ankle joint; (b) picture following wound debridement and planning of a lateral supramalleolar flap; (c) lateral supramalleolar flap was elevated on day 2 following debridement; (d) lateral supramalleolar flap delay performed; (e) flap inset was completed on day 4 following debridement

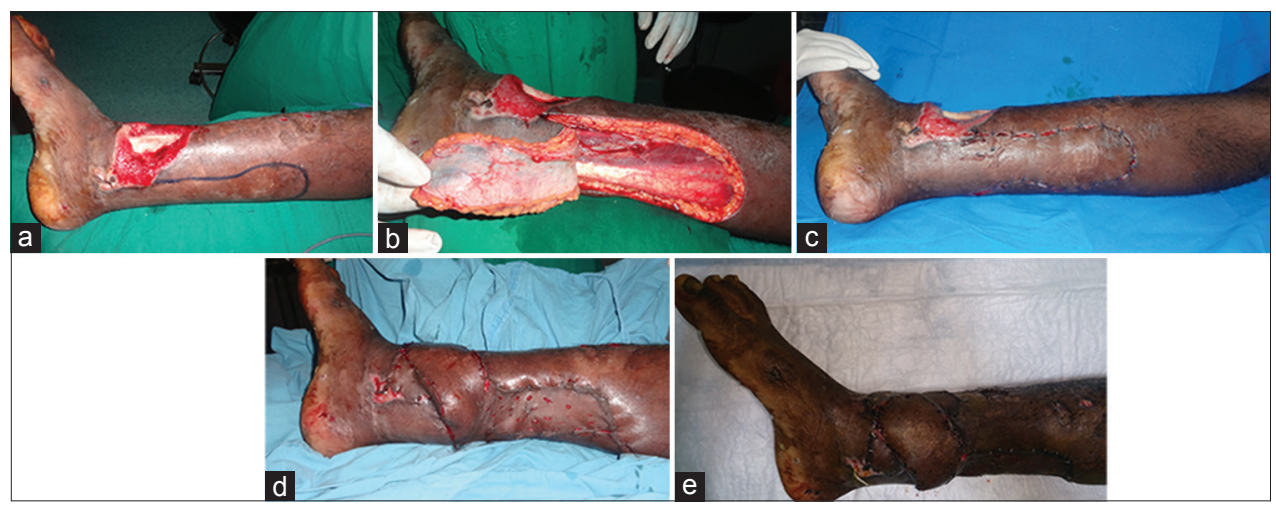

Figure 3: (a) Posttraumatic soft tissue defect exposing the lower third of the tibia; (b) distally-based posterior tibial artery perforator plus flap elevated; (c) distally-based posterior tibial artery perforator plus flap delay performed; (d) flap inset was completed on day 4 following debridement; (e) late postoperative picture of flap

based on the peroneal or posterior tibial artery perforators are frequently used for reconstruction of defects in this region. ${ }^{\mid 1-3]}$ These flaps may be harvested as fasciocutaneous, islanded fasciocutaneous, adipofascial, or propeller flaps, or may be harvested as delayed extended flaps.

One common complication encountered during the utilization of distally-based flaps for such defects is venous congestion, which may result in failure at the distal aspect of the flap, which may be covering a critical region of the defect. Causes for venous congestion include compression of the pedicle due to poor elasticity of the skin over the roof of the tunnel in island flaps, valvular incompetence, edema at the pedicle region, compartment syndrome, and compression of the pedicle by hematoma. 


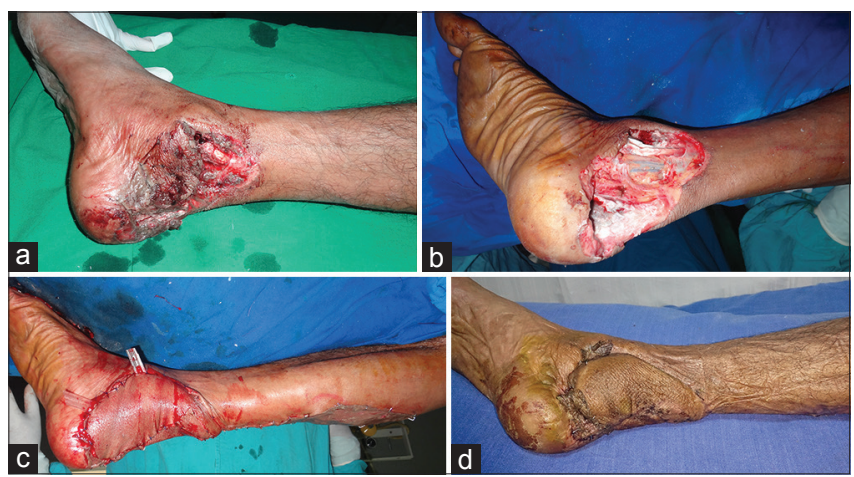

Figure 4: (a) Posttraumatic soft tissue defect with exposure of the medial malleolus and calcaneus; (b) picture following wound debridement; (c) reverse sural artery flap cover performed; (d) tip necrosis of the reverse sural flap covered with a skin graft

Almeida et al. ${ }^{[3]}$ experienced partial flap necrosis (22.1\%), total flap necrosis $(4.2 \%)$, infection $(8.5 \%)$ and venous congestion $(4.1 \%)$ in a total of 71 cases of transferred reverse sural flaps. Zayed et al. ${ }^{[6]}$ experienced venous congestion in five out of 25 cases of lateral supramalleolar flap coverage. Voche et al. ${ }^{[2]}$ reported venous congestion and partial flap necrosis (5-30\%) in 41 cases of a lateral supramalleolar flap used for ankle and foot defects. Kneser et al. ${ }^{[4]}$ suggested a delayed neurofasicocutaneous sural flap, which is initially completely elevated and then fixed again at the donor site using running sutures for 7-15 days. After confirming the flap's survival, the flap is raised again and transposed into the soft tissue defect. This delay procedure could be an alternative to increase the reliability and viability of the distally-based fasciocutaneous flap. However, delay procedures are not feasible in every patient as they require a significant time delay for coverage of vital structures. Ulkuir et al. ${ }^{\text {7] }}$ demonstrated the usefulness of HBO treatment during the delay period of the flap which can lessen the time period required for delay, and which can also increase the effect of flap delay. This technique of reducing the delay period could well be utilized in the reduction of the duration of flap transfer in flap delay procedures. In addition, HBO therapy helps to prepare the recipient and donor areas, and the flap to be transferred during the delay period. There appears to be no harm in administrating $\mathrm{HBO}$ therapy during the delay period, as it reduces the edema of the delayed tissue and provides an optimal outcome following transfer.

In our center, $\mathrm{HBO}$ is administered in a monoplace chamber in which a single patient is placed in a chamber, which is then pressurized with $100 \%$ oxygen. Vasoconstriction reduces edema and tissue swelling while ensuring adequate oxygen delivery and is thus useful in acute trauma wounds as well as in delayed flaps. Hyperoxygenation causes immune stimulation by restoring white blood cell function and enhancing their phagocytic capabilities and neo-vascularization in hypoxic areas by augmenting fibroblastic activity and capillary growth. ${ }^{[8]}$ Adequate shock management, debridement and repair of soft tissues, and stabilization of bony elements are of paramount importance. HBO therapy as an adjunct should be administered as early as possible to minimize the frequency and extent of tissue necrosis, reduce edema, control infection, support healing and prevent reperfusion injury. ${ }^{[9]}$

A recent retrospective analysis of 70 consecutive sural flaps reported a complication rate of 59\% (41 of 70 flaps), with complete necrosis in 19\% flaps and partial necrosis in $17 \% .^{[10]}$ In a series of lateral supramalleolar flaps by Ehab et al. ${ }^{[6]}$ a total of 5 patients (20\%) suffered complications out of 25 patients. Two cases were managed conservatively, 2 cases required revision with suturing, and 1 case required alternative flap coverage. Kang et al. ${ }^{[1]}$ experienced 4 patients with partial necrosis (30\%) among 13 patients where distally-based sural artery and lateral supramalleolar flaps had been utilized for soft tissue defects of the leg and foot. We noted complete flap survival in patients who received HBO with flap delay in spite of their associated co-morbidities. In the transfer group without HBO treatments, 5 patients of 11 (45.4\%) experienced flap tip necrosis, and 1 patient had partial flap loss.

At our institution, we have developed a strategy to successfully manage patients with defects of the lower third of the leg and foot using a combined approach that maximizes tissue perfusion and oxygenation, allowing for optimal surgical correction of such injuries. Our treatment algorithm begins with early surgical debridement and initiation of HBO therapy. Combination of the modalities allows preservation of marginal tissue, prevention of extension of ischemia, reduction of tissue edema and congestion, and maximum preservation of the transferred distally-based flap. In our series, no complications were noted in patients treated with this approach. However, several cases of the flap tip or partial necrosis were noted in patients who received direct flap transfer. In this series, flap delay procedures were scheduled based on various factors including severity of injury, time of referral, co-morbid conditions, patient age, reach of the flap, patient toleration of use of the chamber, and affordability of treatment. However, additional studies are required to determine any additional indications, as well as the optimal timing and dosage of HBO therapy for such procedures. The patients in the current series did not experience the common side effects of HBO therapy such as aural or pulmonary barotrauma or a transient reversible myopia. Optimal usage of HBO therapy may reduce the duration of flap delay and increase the effect of flap delay procedure, helping to an optimal outcome for the transferred tissue.

In conclusion, distally-based flaps provide effective coverage of variable sized soft tissue defects of the lower third of leg, ankle and foot following trauma. Adjunctive HBO therapy should be considered when possible for improved flap survival and optimal surgical outcomes.

\section{REFERENCES}

I. Demiri E, Foroglou P, Dionyssiou D, Antoniou A, Kakas P, Pavlidis L, Lazaridis L. Our experience with the lateral supramalleolar island flap for reconstruction of the distal leg and foot: a review of 20 cases. Scand J Plast Reconstr Surg Hand Surg 2006;40:106-10. 
2. Voche P, Merle M, Stussi JD. The lateral supramalleolar flap: experience with 4I flaps. Ann Plast Surg 2005;54:49-54.

3. Almeida MF, da Costa PR, Okawa RY. Reverse-flow island sural flap. Plast Reconstr Surg 2002; 109:583-91.

4. Kneser U, Bach AD, Polykandriotis E, Kopp J, Horch RE. Delayed reverse sural flap for staged reconstruction of the foot and lower leg. Plast Reconstr Surg 2005; 1 16:1910-7.

5. Mehrotra S. Perforator plus flaps: optimizing results while preserving function and esthesis. Indian J Plast Surg 2010;43:141-8.

6. Zayed EF. Lateral supramalleolar flap for reconstruction of the distal leg and foot, clinical experience with 25 cases. Egypt J Plast Reconstr Surg 201।;35:279-86.

7. Ulkür E, Karagoz H, Ergun O, Celikoz B, Yildiz S, Yildirim S. The effect of hyperbaric oxygen therapy on the delay procedure. Plast Reconstr Surg 2007; । 1 9:86-94.

8. Bhutani S, Vishwanath G. Hyperbaric oxygen and wound healing. Indian J Plast Surg 20I2;45:3।6-24.
9. Kemmer A. Crush injury and other traumatic ischemia. In: Mathieu D, editor. Handbook on Hyperbaric Medicine. Netherlands: Springer; 2006. p. 3II-2.

10. Baumeister SP, Spierer R, Erdmann D, Sweis R, Levin LS, Germann GK. A realistic complication analysis of 70 sural artery flaps in a multimorbid patient group. Plast Reconstr Surg 2003; I I2:129-40.

II. Kang HG, Kim JH, Cho HS, Han I, Oh JH, Kim HS. Soft tissue reconstruction of the foot using the distally based island pedicle flap after resection of malignant melanoma. Clin Orthop Surg 2010;2:244-9.

How to cite this article: Korambayil PM, Ambookan PV. Hyperbaric oxygen therapy and surgical delay improve flap survival of reverse pedicle flaps for lower third leg and foot reconstruction. Plast Aesthet Res 2015;2:130-7.

Source of Support: Nil, Conflict of Interest: None declared.

Received: 07-12-2014; Accepted: 29-01-2015 